\title{
CARPOOLING: A MULTI-AGENT SIMULATION IN NETLOGO
}

\author{
Marcelo Armendáriz, Juan C. \\ Burguillo, Ana Peleteiro \\ Dep. of Telematic Engineering \\ University of Vigo \\ 36310-Vigo (Spain) \\ E-mail: J.C.Burguillo@det.uvigo.es \\ E-mail: apeleteiro@det.uvigo.es
}

\author{
Gérald Arnould, Djamel Khadraoui \\ CRP Henri Tudor \\ 29,. Avenue John F. Kennedy \\ L-1855 Kirchberg (Luxembourg) \\ E-mail: Gerald.Arnould@tudor.lu \\ E-mail: djamel.khadraoui@tudor.lu
}

\section{KEYWORDS \\ Carpooling, Mobility, Simulation, Netlogo.}

\begin{abstract}
The WiSafeCar (Wireless Traffic Safety Network between Cars) project aims at increasing the performance and reliability of the wireless transport and to provide traffic safety improvements. Within the context of this project, we have designed a Dynamic Carpooling System that will optimize the transport utilization by the ride sharing among people who usually cover the same route. An initial prototype of the system has been developed by using NetLogo. The information obtained from this simulator will be used to study the functioning of the clearing services, the current business models and to propose new ones. The first results seem encouraging, and the users have many economical advantages thanks to the sharing of costs which allows the individuals to retrench expenses and to contribute to the use of green technologies.
\end{abstract}

\section{INTRODUCTION}

In the past few years, governments, industry and academia have been more and more interested in exploring road safety and other type of vehicular applications by connecting car-to-infrastructure (C2I) and car-to-car $(\mathrm{C} 2 \mathrm{C})$ with several wireless broadband technologies, such as $2 \mathrm{G} / 3 \mathrm{G}$, Mobile WiMAX and WiFi. So far a variety of safety related and other vehicular applications have been developed and wireless technologies for mobility have been studied in several research projects. The main target areas of those activities are/have been: 1) wireless technology research for high-speed vehiculars, 2) in-vehicle control device and sensing technology research, and 3) real-time vehicular application research. For example, the CELTIC's CARLINK project (http://carlink.lcc.uma.es) developed an intelligent traffic service platform based on hybrid wireless networking targeted at vehicular networking research for seamless C2I and C2C communications, and several intelligent multi-modal transport services based on real-time measurements from smart car sensors.
The Car2Car Communication Consortium (http://www.car-to-car.org) is working on extensive standardisation of vehicle networking systems. However, some critical issues which lay between wireless technologies and services have not been solved nor addressed by any of those activities, for instance, it is important to consider how data (content) should be handled in the most efficient and reliable manner. This efficiency is important for services used in a high-speed vehicular environment where the frequently-changing topology makes the real deployment more challenging.

The CRP Henri Tudor from Luxembourg and several institutions from Europe and Asia have been involved into a new initiative called WiSafeCar (Wireless Traffic Safety Network between Cars, http://www.wisafecar.com) as a continuation of the CARLINK project. The WiSafeCar platform is the linking point of several services and physical infrastructure, hosting roadside units and furthermore serving vehicles and mobile units acting as customers of the platform. This project provides the costumers with new telematic services for vehicles regarding meteorological, traffic information, $\mathrm{C} 2 \mathrm{C}$ communications and multi modal transport management services.

In this paper, we present the Dynamic Carpooling System, integrated within WiSafeCar, that will optimize the transport utilization among people who usually cover the same route. Carpooling is a simple way for individuals to take part in the climate change challenge while saving money, reducing congestion and conserving energy along the way. This system, very useful for commuters that live near each other and share a common destination, can be very advantageous for occasional travellers too. The idea is to share a car depending on the destination of the passengers, so they can save costs, and it also helps to reduce pollution and traffic jams.

Classical initiatives for carpooling did not succeed due to the lack of flexibility for the user who demands a fast and safe service at anytime and anywhere. Usually, classical approaches were only suitable for static situations where the travel time and the destination 
have been planned before (Keenan and Brodiey, 2000; Psannis et al., 2006; Kelley, 2007; Yew et al., 2008; Ferreira et al., 2009). This is why there is a lot of effort to try to provide these services in a fast changing mobile environment (Correia and Viegas, 2009).

The rest of the paper is organized as follows. First we provide an introduction to Urban Traffic Simulations in Netlogo. Then we introduce the Dynamic Carpooling Simulator. Afterwards, we describe the results obtained by the simulator, and finally we present the conclusions and future work.

\section{URBAN TRAFFIC SIMULATION IN NETLOGO}

The Dynamic Carpooling Simulator, within WiSafeCar, was implemented in NetLogo (http://ccl.northwestern.edu/netlogo/), a multi-agent programming language and modelling environment for simulating natural and social phenomena.

Urban Traffic Simulation starts in Netlogo with the Gridlock model by (Wilensky and Stroup, 1999). Several enhancements have been done based on this initial model included in NetLogo distribution. In (Gershenson 2005) we have an extended version of the Gridlock model that consists of an abstract traffic grid with intersections between cyclic single-lane arteries of two types: vertical or horizontal. Cars only flow in a straight line, either eastbound or southbound. Each crossroad has traffic lights which allow traffic flow in only one of the arteries, which intersect it with a green light. Yellow or red lights stop the traffic. The light sequence for a given artery is green-yellow-red-green.

Based on this enhanced version of the Gridlock model, the GTI group from the University of Vigo has carried out new extensions improving the MAS simulator in order to make it more realistic (Burguillo et al., 2009). This simulator removed the torus and improved the car movements in order to let them drive in four directions: north, east, south and west. We created a by-pass road to improve traffic, which is the outermost in the scenario. Regarding the structure of the map and the roads, this new version has the possibility of bidirectional roads and roads with two lanes in the same direction, controlled with a slider in the NetLogo interface. Besides, an algorithm to avoid deadlocks at the intersections was implemented.

Finally, this new simulator also included a new functionality where some drivers could be considered as commuters. A commuter car drives in the city and stops whenever it has arrived to its destination. Afterwards, it drives back home. In this way, the origin and destination are exchanged at each stage.

\section{THE DYNAMIC CARPOOLING SIMULATOR}

A new version of the simulator has been developed in a collaboration between the GTI group from the University of Vigo and SSI of the CRP Henri Tudor in Luxembourg. As the simulator already developed by the GTI was able to manage an urban traffic area, modeling the cars as agents, and implementing several traffic lights mechanisms; in the new version presented in this paper (see Figure 1) we mainly add the dynamic carpooling functionality. But, we also enhance some of the previous functionalities of the multiagent system in order to get a more realistic scenario.

Since the Dynamic Carpooling System design had been previously carried out, the goal of the simulator was to evaluate how it works with different parameter settings and to gather information related to the trips in order to analyse current business models and/or to develop new ones. The Dynamic Carpooling Simulator includes a set of new features, which are described next.

\section{Car Creation Improvements}

With this new approach, cars have new attributes and targets to follow. Cars have an identification number, driverID, which distinguish them from the other cars, in terms of passengers association, in order to do the corresponding compensation at the end of each trip. Each car keeps count of the distance covered during the itinerary, and the time spent from the moment it was started until the destination was reached.

The simulator interface provides the user with a slider, with values between $0 \%$ and $100 \%$, to choose the probability that a car belongs to a carpooling provider. In case a car is part of a carpooling system, they must belong to a specific provider (A, B or C) which is also determined according to the corresponding parameters.

The system will try to match these carpoolers with the users who have requested the service and thus a new itinerary will be set to them. The fact that a car is part of a carpooling system does not guarantee that it will perform a service giving a ride to one or more passengers. In the previous versions, the drivers had an origin and a destination, and those values were definitely unchanged. Now, depending on the number of passengers associated to it, the car has a list of intermediate stops where it picks up or drops clients, before arriving to the destination.

The number of passengers inside each car can be selected using a slider. The minimum value is 0 and the maximum is 4 . When a passenger is assigned to a car, his identification is stored in the passengers-list of the car, and when the maximum number of passengers in the car is reached, the vehicle will not be available. 


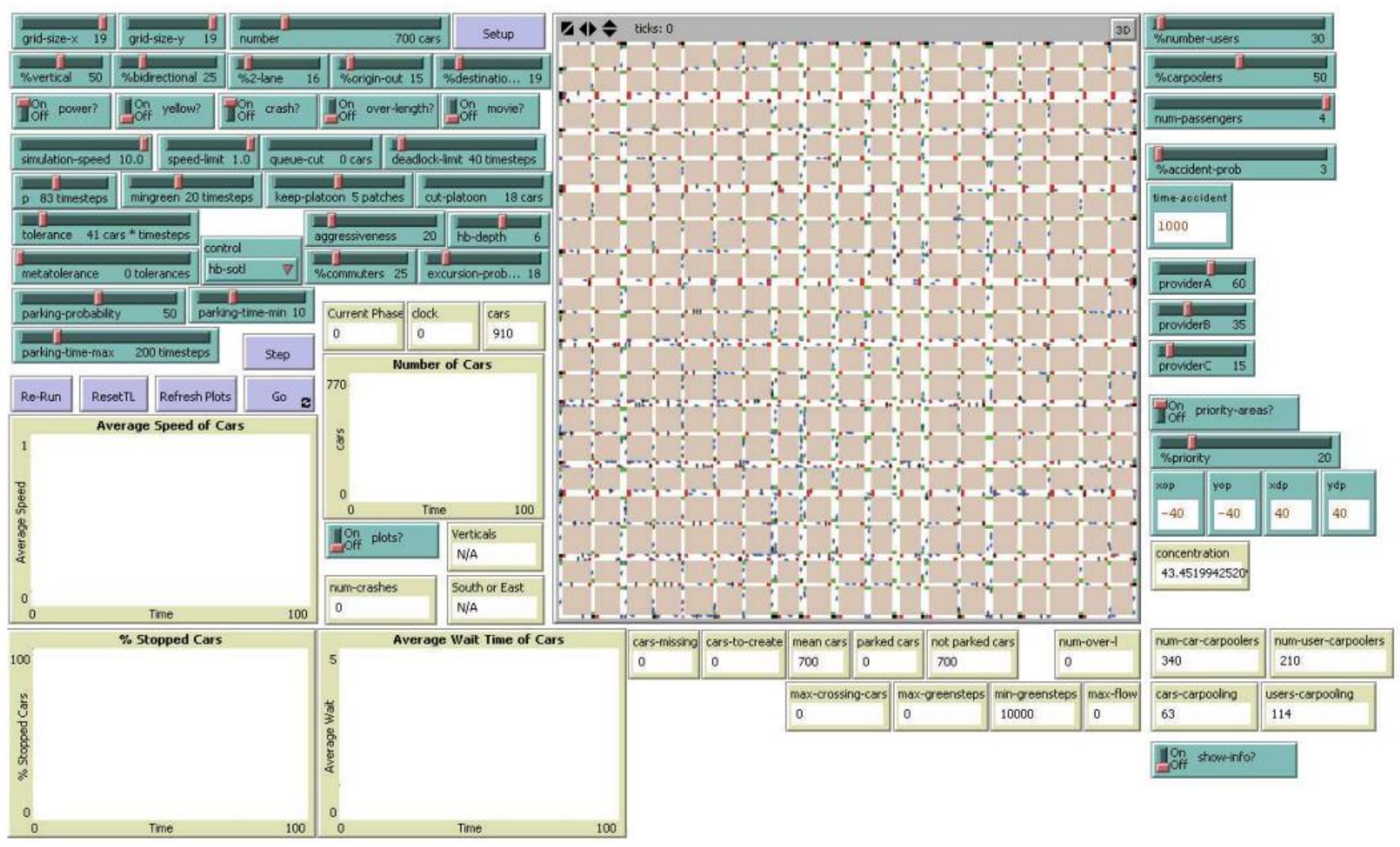

Figure 1: Snapshot of the Dynamic Carpooling Service Simulator

\section{User Creation}

Users also have an identification number, userID, with which the driver is able to authenticate himself in the carpooling system. In order to identify if a user has been served or not, a happy or sad face appears on his location. After a random period of time these users disappear and new ones are created in other city location. The number of carpooling potential users is easily adjusted by using the corresponding slider.

As the users are actually clients, they must belong to a carpooling provider, and the system will try to match them with the most suitable driver from such provider.

Once a passenger gets into a car, his userID is added to the list of 'present-passengers'. Each passenger keeps count of the distance covered, and the time spent to do the trip. Insomuch we are talking about services and providers, we also talk about business. A compensation method for a passenger may depend on the number of passengers he is sharing with. For this reason each user keeps count of the time and distance covered sharing with one, two or three passengers, or travelling alone.

\section{Matching algorithm}

An interesting functionality included in the carpooling simulation is the matching algorithm, which is in charge of finding a suitable driver for the carpooling passengers.
First of all, when a trip is requested by a user, the system seeks the drivers that are travelling in the same direction that the passenger wants to follow. Once it has the list of possible drivers, the matching algorithm compares the origin and destination of the passenger to the drivers' paths. If a driver whose itinerary contains the client's trip is found, the matching algorithm checks if the car has available places and if so, the user will be labelled as matched. The driver assigned to this user has to add two more intermediate stops in his destinations-list, the pickup location and the drop point. Likewise he adds the userID of the passenger to the passengers-list. If there is no driver who fits the request, the user will show a sad face and disappear after a period of time. A new one will be created afterwards in another city point.

\section{High populated areas}

Thinking about a real situation in a city, is quite often to find places more populated than others, and also destinations more demanded, as the people are not evenly distributed. For instance, most of the people tend to concentrate in the centre of a city rather than the outskirts. It is also very common that there are certain areas where most of the people go to work, such as business, commercial or industrial zones.

For this reason, and aiming to simulate a more realistic situation, we have developed a new functionality where we can choose the area of the map were the population density is higher by entering the coordinates of the 
central point of this overpopulated region. The radius of this area and the level of concentration depend on the number of people belonging to the city and the percentage of the people who live in such area.

Likewise, we can simulate the business area of the city where the majority of the people demand to go. The mechanism is the same: we select the central point by entering the coordinates, and the passengers will request to go to that place with a certain probability. By adjusting the slider shown in Figure 2, we can decide what percentage of the population belongs to these zones. In the input boxes labelled as 'xop' and 'yop' we enter de coordinates of the patch which is the central point of the most populated area. With the boxes labelled as ' $x d p$ ' and 'ydp' we can set the coordinates of the central point of the most demanded zone. Finally, in the box named 'concentration', the level of concentration of the population is shown.

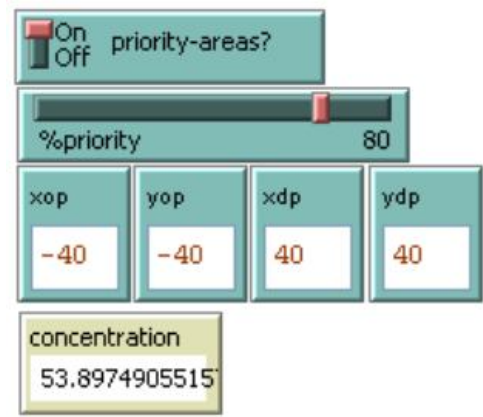

Figure 2: Controllers for managing the concentration of the population

Figure 3 shows a situation where the $80 \%$ of the people is located in the same region. Given the central patch of the map as the coordinate $(0,0)$, we can see how the bluish cars are concentrated in the southwest of the city, all of them distributed around the patch $(-40,-40)$ as was shown in the figure 2 .

\section{Accident avoidance}

Another typical event regarding the urban traffic are the traffic jams caused by accidents. As usual, accidents can block the streets and do not allow the movement of cars. In order to avoid this situation we make the car to choose an alternative route.

As it was implemented in the previous versions of the simulator, a crash can happen when two cars meet in the same point. In this version, the accident can only happen at an intersection, and we add the possibility that a street can get blocked. In case of an accident, and with a given probability, the street may get blocked during a period of time. Since the accidents happen at a concrete intersection, which involves two streets, the stretches of roads leading from such intersection to the adjacent intersections will not be available to drive through them. Since we assumed that the drivers have updated information of the incidents on the road, they are able to change their paths in order to avoid the blocked streets. The cars, which were in those streets when the accident happened, remain stopped, waiting until the end of the blocking period.

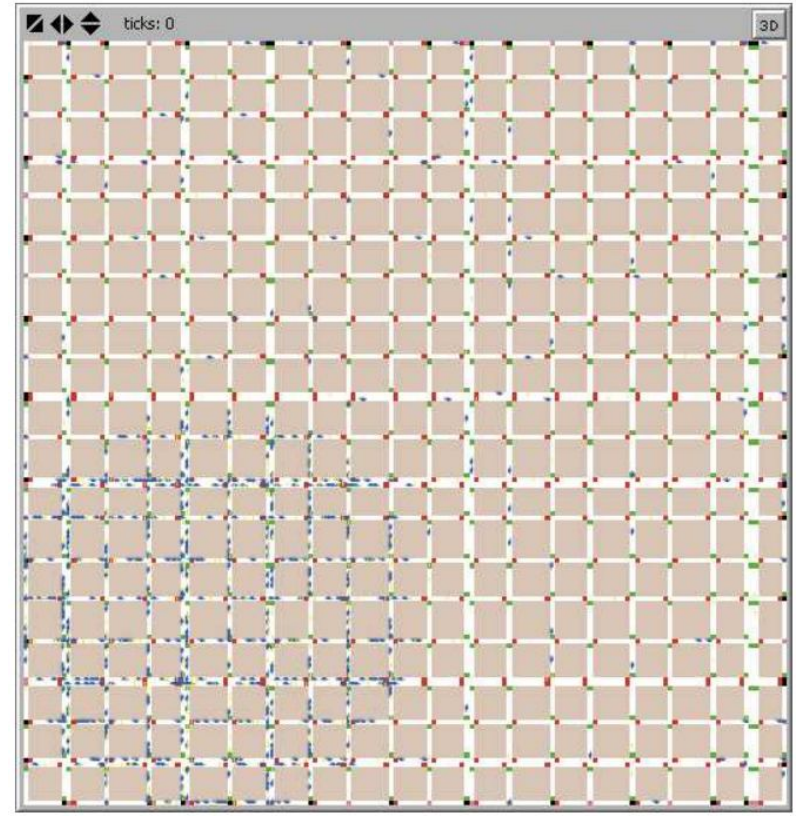

Figure 3: Snapshot with a high level concentration area

We can determine in advance the blocking probability in case of accident by using a slider with values ranging from $0 \%$ to $100 \%$. Besides, we can also enter the time a street can be blocked by using an input box.

\section{Data storage}

The goal of the simulation is to store all the information related to the itineraries such as the distance covered, the time spent, the number of people who share the car during the trip, etc.

At the end of each trip, a new file named with the user identifier, is created. We consider a trip as every route covered by one passenger since he gets into the car until he gets out. Therefore, the itinerary of one driver can contain several single trips.

As we stated before, users gather data at every step of the simulation by keeping count of the distance they have already covered. In the same way they count the time spent during their itineraries bearing in mind the passengers they are sharing with.

The mentioned log file contains all the information that is necessary to proceed with the corresponding compensation method. The contents of the file is described in Table 1. 
Table 1: Trip data gathered by the carpooling system

\begin{tabular}{ll}
\hline Data & Description \\
\hline UserID & User Identifier \\
\hline UserProvider & User Provider \\
\hline DriverID & Driver Identifier \\
\hline DriverProvider & Driver Provider \\
\hline TripStartTime & Start time of the trip \\
\hline TripDuration & Duration of the trip \\
\hline DistanceSharedBy1 & Distance covered by the car with 1 passenger \\
\hline DistanceSharedBy2 & Distance covered by the car with 2 passenger \\
\hline DistanceSharedBy3 & Distance covered by the car with 3 passenger \\
\hline DistanceSharedBy4 & Distance covered by the car with 4 passenger \\
\hline TimeSharedBy1 & Time covered by the car with 1 passenger \\
\hline TimeSharedBy2 & Time covered by the car with 2 passenger \\
\hline TimeSharedBy3 & Time covered by the car with 3 passenger \\
\hline TimeSharedBy4 & Time covered by the car with 4 passenger \\
\hline
\end{tabular}

\section{System outputs}

Apart from the files provided by the simulator and the plots implemented before, the system produces some extra data. We have several output boxes with log information that can be displayed depending on whether the switch is on or off (see Figure 4).

\begin{tabular}{|c|c|}
\hline num-car-carpoolers & num-user-carpoolers \\
\hline 340 & 210 \\
\hline cars-carpooling & users-carpooling \\
\hline 63 & 114 \\
\hline
\end{tabular}

Figure 4: Monitor boxes and switch.

In the 'num-car-carpoolers' box it is shown the number of cars that are labelled as carpoolers, which means they are able to provide the service. The box 'carscarpooling' keeps count of the number of cars that are currently giving a ride to, at least, one passenger. The monitor box labelled as 'num-user-carpoolers' shows the number of users who have requested the service. Finally, the monitor called 'users-carpooling' displays the number of users who have been already matched with a driver. They can be travelling or waiting for the car to pick them up.

During the simulation we can see several data displayed in the log window. Once a passenger is matched, his identification number and the identifier of the driver who will provide him the service will be shown. Then, when the driver picks him up, another notification will be written in this log, and the same happens when the passenger reaches his destination.

In case of an accident, the coordinates of the patch involved will be displayed on the screen and will turn orange in the map. In the same way the blocked streets will become pink.
If a user cannot be matched, a red sad face will appear on his location, but; if the user has been served, a green smiley face will be shown at the destination.

\section{RESULTS}

Since we assumed that the first step in providing the carpooling service to a client is to ensure that he can afford the trip cost, we need to calculate the amount of credit the user must have in his account. With the aim of evaluating the clearing service, proposed for the Dynamic Carpooling System, and in order to get a fair estimate of the cost of the trips, we have adapted the simulator to obtain data related to the ideal route a driver could cover in terms of traffic and accidents. Using this results we will be able to compare a normal trip, where traffic jams are common, with an ideal one by comparing the time spent during the route, and the distance covered in both situations.

We stablished 15 scenarios with several parameters and we simulated them in both normal and ideal conditions. Regarding the ideal trip, we have assumed no other cars in the map, and the absence of accidents. The rest of the conditions remained unchanged.

In this context, we fixed the value of the amout of cars that the belong to the system as the $50 \%$ of the total number of cars for each scenario. The number of passengers inside a car was 3 and the probability of accident stablished was $1 \%$. The size of the map and the distribution of the lanes was the same for all the simulations, as well as the origin and destination of each car and passenger. We considered the centre of a city, as a manhattan like scenario, with $2 \mathrm{~km} \times 2 \mathrm{~km}=$ $4 \mathrm{~km}^{2}$. The main numbers obtained as results from the simulations are shown in Table 2.

As we can see in the table, the ideal estimation of the distance covered, and the time spent during the route are mostly the same, in normal conditions than in the ideal ones, for a low number of cars. The values start to differ when the number of cars is increased. If we think of a real situation, the same thing occurs if we compare the traffic during the rush hours and during the night, so we have to estimate the suitable cost of the trip depending on the time of the day.

The time spent during a trip and the distance covered by the user in normal conditions is increased on average a $6.6 \%$ and $8.4 \%$, respectively, with respect to the ideal situation, when the number of cars change from 300 to 500 . The same thing happens when the number of cars is fixed as 1000 . The duration of the trip is increased a $6 \%$ and the distance covered a $38.8 \%$. 
It is important to note that, in the conditions we have evaluated the system, the number of users served never exceeds the $55 \%$. This maximum is reached when the number of drivers is five times greater than the number of users waiting to be served, and when the $50 \%$ of cars belong to the carpooling system. The performance improves with the increasing of the population.

Table 2: Simulation results for several combinations of input data

\begin{tabular}{|c|c|c|c|c|c|c|c|c|}
\hline $\mathbf{N}$ & Number of Cars & Users & Users Served & Users Not served & Ideal Distance & Ideal Time & $\begin{array}{l}\text { Normal } \\
\text { Distance }\end{array}$ & $\begin{array}{c}\text { Normal } \\
\text { Time }\end{array}$ \\
\hline 1 & 100 & 40 & 8 & 32 & 117.7 & 271.75 & 119.2 & 278.38 \\
\hline 2 & 100 & 100 & 16 & 84 & 252.58 & 545.50 & 256.63 & 562.19 \\
\hline 3 & 200 & 40 & 18 & 22 & 124.38 & 284.83 & 124.50 & 291.11 \\
\hline 4 & 200 & 100 & 26 & 74 & 175.09 & 389.69 & 180.39 & 408.35 \\
\hline 5 & 300 & 40 & 18 & 22 & 144.9 & 339.40 & 145.68 & 344.61 \\
\hline 6 & 300 & 80 & 38 & 42 & 197.67 & 480.29 & 197.73 & 480.30 \\
\hline 7 & 300 & 150 & 48 & 102 & 180.90 & 428.35 & 182.56 & 432.12 \\
\hline 8 & 300 & 300 & 86 & 214 & 151.68 & 341.91 & 153.93 & 356.92 \\
\hline 9 & 500 & 100 & 49 & 51 & 152.73 & 355.39 & 178.08 & 399.48 \\
\hline 10 & 500 & 250 & 106 & 144 & 199.48 & 445.77 & 200.23 & 473.44 \\
\hline 11 & 500 & 400 & 150 & 250 & 205.22 & 458.79 & 209.22 & 489.85 \\
\hline 12 & 500 & 500 & 184 & 316 & 205.39 & 450.22 & 221.05 & 487.98 \\
\hline 13 & 1000 & 200 & 110 & 90 & 181.23 & 428.89 & 191.74 & 573.78 \\
\hline 14 & 1000 & 500 & 236 & 264 & 201.62 & 469.61 & 217.74 & 654.07 \\
\hline 15 & 1000 & 1000 & 391 & 609 & 210.56 & 471.08 & 220.01 & 675.45 \\
\hline
\end{tabular}

\section{CONCLUSIONS}

In this paper we have introduced the WisafeCar platform. WiSafeCar aims at increasing performance and reliability of the transport, and wireless services for traffic safety improvements. WiSafeCar also provides the costumers with new telematic services for vehicles regarding meteorological, traffic information, and consider the use of green technologies to reduce traffic congestion and energy expenditure.

In the context of transport management services, we have designed a Dynamic Carpooling System that will optimize the transport utilization by the ride sharing among people who usually cover the same route. An initial prototype of the system has been developed by using NetLogo an extending an Urban Traffic MAS simulator. The information obtained will be used to study the functioning of the clearing services, and to propose new business models.

The first results seem encouraging, and the users have many economical advantages thanks to the sharing of costs which allows the individuals to retrench expenses. The driver should have extra discounts or benefits considered under the business model. We also conclude that this system could have a bigger impact in larger populations than in the smaller ones, and that the amount of drivers available at the carpooling service must be several times greater than the number of users waiting to be served. We also have come to the conclusion that the persistence of a carpooling system can be strongly benefitted by the integration of the public transports services, since many routes cannot be frequently covered by the drivers belonging to the carpooling system alone.

Several works have been carried out during the last years, most of them web based and able to schedule itineraries between passengers; but they can not provide a real time service and track itineraries in order to ensure the security of the users. Our system provides an intelligent matching service along with a smart routing engine that can use real time information (for instance, considering weather and traffic conditions).

Future work will enhance the urban traffic simulator with new functionalities to provide a more realistic scenario. For instance, pedestrian crossing or public transports such as buses or taxis could be added to provide an alternative to car use in some routes. 


\section{ACKNOWLEDGEMENTS}

The authors want to thank their colleagues Pedro S. Rodríguez and Enrique Costa for the collaboration in the development of the traffic simulator in Netlogo. This work was partially supported by the Xunta de Galicia under INCITE Project Number 10 PXIB 322 039 PR.

\section{REFERENCES}

Burguillo, J.C.; Rodriguez, P.; Costa, E and Gil, F. 2009. "History-based Self-Organizing Traffic Lights", Computing and Informatics. Vol. 28, no. 2, 157-168.

Correia, G. and Viegas, J.M. 2009. "A conceptual model for carpooling systems simulation". Journal of Simulation 3, 61-68.

Ferreira J.; Trigo P. and Filipe P. 2009. "Collaborative Car Pooling System". World Academy of Science, Engineering and Technology 54.

Gershenson, C. 2005. "Self-organizing traffic lights". Complex Systems, Vol. 16 (1). 29-53

Keenan, P. and Brodiey S. 2000. "A Prototype Web-based Carpooling System". Americas Conference on Information Systems, AMCIS 2000. Proceedings, Association for Information Systems.

Kelley, K.L. 2007. "Casual Carpooling_Enhanced". Journal of Public Transportation, Vol. 10, No. 4.

Psannis, K.; Hadjinicolaou, M.; and Ishibashi Y. 2006. "Designing a Pervasive Architecture for Car Pooling Services". Advances in computer, information, and systems sciences, and engineering. 211-218.

Wilensky, U. and Stroup, W. 1999. "NetLogo HubNet Gridlock model. Center for Connected Learning and Computer-Based Modeling". Northwestern University, Evanston, IL.

Yew, K.H.; Mustapha, E.E.; Do D.K. and Chen, Y.Y. 2008. "Pervasive Car Pooling System using Push Strategy". International Symposium on Information Technology ITSim. Kuala Lumpur.

\section{AUTHOR BIOGRAPHIES}

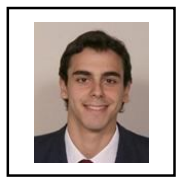

MARCELO ARMENDÁRIZ received the M.Sc. degree in Telecommunication Engineering in 2010 at the University of Vigo, Spain. He is currently enrolled in the Master in Telemedicine and Bioengineering at the Polytechnic University of Madrid. His research interests include neural networks, telematic services, multi-agent systems and the field of bioengineering related to biomedical imaging and medical decision support systems. His e-mail is: m.armendarizealumnos. upm.es

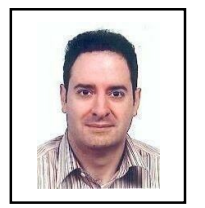

JUAN C. BURGUILLO received the M.Sc. degree in Telecommunication Engineering in 1995, and the Ph.D. degree in Telematics (cum laude) in
2001; both at the University of Vigo, Spain. He is currently an associate professor at the Department of Telematic Engineering at the same university. He has participated in several $R \& D$ projects in the areas of Telecommunications and Software Engineering, and has published more than one hundred papers in journals and conference proceedings. His research interests include game theory, optimization, telematic services, autonomous agents and multi-agent systems. His e-mail address is: jrialedet.uvigo.es and his Web-site is at: http://www.det.uvigo.es/ jrial

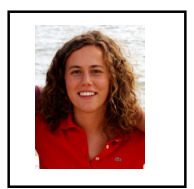

ANA PELETEIRO received the M.Sc. degree in Telecommunication Engineering in 2009, and a M.Sc. degree in Telematics in 2010; both at the University of Vigo, Spain. She is currently a project engineer at the Department of Telematic Engineering at the same university. Her research interests include game theory, selforganization, autonomous agents and multi-agent systems. Her e-mail is: apeleteiro@det.uvigo.es and her Web-site is at: http://wwwgti.det.uvigo.es/ apeleteiro

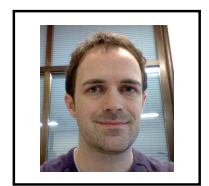

GERALD ARNOULD received his PhD in Microelectronics in 2006 at the University of Metz, France. $\mathrm{He}$ is currently a postdoctoral researcher and project manager at the CRP Henri Tudor, Luxembourg. He has 6 years' experience in the fields of mobile ad-hoc and hybrid networks resulting in several international publications. $\mathrm{He}$ has been working on EU and national research projects (WISAFECAR, CLAIRVOYANT) in the fields of context aware and intelligent mobility. Email: gerald.arnould@tudor.lu

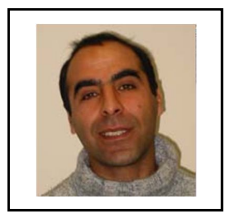

DJAMEL KHADRAOUI, Lead R\&D Manager is coordinating several European projects in ICT for sustainable mobility, intelligent systems and software engineering. He is DC COST member in the domain of ICT and Transport. He is involved in many conferences and workshops. He published a book in coordination with IDEA Group Publishing. He mainly participated and coordinated several previous and current EU projects: MICIE, LINKALL, as project manager, CARLINK, WISAFECAR, RED, and STIMULATE as technical project coordinator. Email: djamel.khadraoui@tudor.lu 